\title{
What's in Your Pocket? Trends in Mobile Apps for Biosurveillance and Decision-Making
}

\author{
Courtney D. Corley*1, Onicio Leal-Neto ${ }^{2}$, Craig Taylor ${ }^{3}$, César Escobar-Viera ${ }^{4}$ and Victor \\ Del Rio Vilas 5
}

${ }^{1}$ Pacific Northwest National Laboratory, Richland, WA, USA; '2Epitrack, Recife, Brazil; ${ }^{3}$ Cojengo, Glasgow, United Kingdom; ${ }^{4}$ University of Florida, Gainesville, FL, USA; ${ }^{5}$ Pan American Health Organization (PAHO), Rio de Janeiro, Brazil

\section{Objective}

To review current trends and issues in the development and use of mobile apps for public health surveillance and decision making in settings with different resource availability and technological development. The panel discussion will address cross-cutting issues of general interest, including timeliness, recruitment, validation, and engagement by presenting innovative examples of apps conceived for various uses in human and animal surveillance.

\section{Introduction}

An increasing number of mobile applications available for download provide biosurveillance capabilities using new and traditional data streams. Biosurveillance apps span a wide range of settings, uses, technologies, and resource capacities that provide health analysts rapid and efficient means of data collection, visualization, and analyses. However, this technological "heaven" is not free from the challenges of traditional biosurveillance applications, namely validation to inform specificity and recruitment and engagement to ensure representativeness. This panel will provide guidance for future development and utility of mobile apps by illustrating how these matters are addressed in field tested mobile applications.

\section{Description}

The panel comprises epidemiologists, data scientists, and technology partners who will address the full application lifecycle of several biosurveillance apps, from the assessment of the specific demand, resource, and technology specifications, to implementation and evaluation. The panel also describes how the apps highlighted navigated their specific setting constraints and the lessons learned in addressing standard biosurveillance issues, namely timeliness, validation, engagement and recruitment. Court Corley's "Data - if we can get it, should we use it?" presentation covers the types of data useful for "mobile health", if they differ from traditional public health data sources, and the issues around using novel open web data sources due to legal and ethical concerns. Onicio Leal-Neto's "Saúde na Copa App - Engaging users with participatory surveillance" presentation discusses methods for actively and passively engaging with mobile users to promote health surveillance and decision apps during mass gatherings. "MozzieMap, a tool to enhance the decision-making process for vector abatement programs" by Cesar Escobar-Viera describes mobile applications to improve tracking and prediction of changes in mosquito population for vector-borne diseases. Finally, Craig Taylor describes "VetAfrica: a mobile application developed by Cojengo supported by the Microsoft 4Afrika initiative" to address the lack of access to experienced veterinary assistance on the ground, accurate real time disease surveillance data, and misdiagnosis of livestock diseases in Kenya.

The subsequent discussion will evaluate how the new systems improved biosurveillance and their impact on the health of the targeted populations, as well as their contribution to the development of the "mobile health" field. The panel provides the basis for an evaluation framework and constitutes a repository of practical advice for the development and implementation of biosurveillance apps. Throughout the panel, the moderator will question the audience via a newly developed web app about their collective experience with biosurveillance apps.

\section{Audience Engagement}

The panel will be presented with the novel online software tool Prezi so that the audience can engage with the content during the panel. For question asking and prioritization we will use multiple methods. First, we will allow the auidence to SMS the moderator their questions, Secondly, we will employ a webapp to be developed collaboratively by the panelists to directly engage the audience in gathering and sharing real-time feedback. In order to be dynamic to the audience needs, we provide the audience a hashtag to use when chatting about the panel in social media and the panelists and moderator will monitor the back channel conversations and adjust the discussion appropriately.

\section{Keywords}

mobile applications; biosurveillance; engagement; timeliness

\section{Acknowledgments}

The MozzieMap app was supported by a contract from the U.S. Defense Threat Reduction Agency (DTRA), Joint Science and Technology Office for Chemical and Biological Defense under project numbers CB4045 to the Pacific Northwest National Laboratory (PNNL). PNNL is operated by Battelle for the U.S. Department of Energy under contract DE- ACO576RLO 1830. The Saúde na Copa App was funded by the Skoll Global Threats Fund.

\section{*Courtney D. Corley}

E-mail: court@pnnl.gov 